Currently the hospital website displays live wait times for the adult ED but not for paediatric ED (PED). Seeing as the PED works as a separate entity to adult ED, it beckons the question, 'Is there a need for live PED wait times to be displayed to the public?' with the aim to introduce a service improvement to provide the information if required.

Methods This audit comprised of a mixed methods analysis. The audit recruited accompanying adults of child attendees at the hospital's PED. A questionnaire discussed the use of any other healthcare services and any existing knowledge of current wait times. Analysis included descriptive statistics and cross tabulation with $\chi 2$ test of independence.

Results Sixty participants completed the questionnaire. 53.2\% had not used any other healthcare service before arrival. 93.5\% did not know the wait time before attending and $77.4 \%$ answered it would be useful to know. More than $60 \%$ said that had they known that the website was providing information on wait times they would have checked it before coming to the ED. The association between displaying wait times online and checking the hospital website before attending was significant, $\chi 2(1, \mathrm{~N}=61)=6.18, \mathrm{P}=0.01$.

Conclusion This audit recognised the need to add PED live waiting times onto the hospital website as a service improvement.

The advantages to knowing about current PED waiting times include:

- Choosing to attend ED if the problem was urgent, or seek help from other services

- Reduced attendance reduces the number of breeches and pressure on the department

- Happier patients who at least know what to expect before attending

- Incorporation of live wait times on the hospital website for the PED is currently being designed.

\section{G249(P) ABSTRACT WITHDRAWN}

\section{G250(P) A RETROSPECTIVE STUDY EXAMINING THE EFFECTIVENESS OF DIFFERENT DEXAMETHASONE DOSES IN CHILDREN PRESENTING TO EMERGENCY DEPARTMENTS IN THE NORTHWEST}

${ }^{1} \mathrm{CE}$ King, ${ }^{2} \mathrm{CW}$ Wong. 'Department of Surgery, Liverpool University Hospital Trust, Liverpool, UK; ${ }^{2}$ Emergency Department, Warrington Hospital, Liverpool, UK

10.1136/archdischild-2020-rcpch.216

Background Croup is a common childhood illness, three per cent of children are diagnosed each year. It is usually a selflimiting illness; however, it creates a large burden on the healthcare system particular emergency departments. The use of steroids has been shown to improve clinical symptoms in children when compared to a placebo. The current guidelines advise the use of dexamethasone, with dosage having recently changed from $0.6 \mathrm{mg} / \mathrm{kg}$ to $0.15 \mathrm{mg} / \mathrm{kg}$. However, not all emergency departments have updated their local protocols.

Aim The aim of this study was to examine retrospective data from paediatric emergency departments in the North West of England examining the efficacy of current dexamethasone doses used in the management of croup in the different departments.
Methods This was a multicentre retrospective chart review conducted in paediatric departments in both secondary and tertiary centres in the North West of England. Data was collected between November 2017 to December 2018, with patients aged between $0-18$ years eligible to be included. Eligible patients had to match the inclusion criteria of having a clinical diagnosis of croup. The primary outcome was reattendance to the emergency department with the same aetiology.

Results There were three sites that submitted data with a total of 175 patients, of these 170 had received a dose of dexamethasone in the emergency department. Croup severity was classified in 128 of patients, with the $78 \%$ being classified as mild croup. Of the patients given dexamethasone, 44\% were given a dose of $0.15 \mathrm{mg} / \mathrm{kg}$ with seven patients reattending, $12 \%$ had $0.3 \mathrm{mg} / \mathrm{kg}$ with three reattendances, and $44 \%$ were prescribed $0.6 \mathrm{mg} / \mathrm{kg}$ with three reattending. There was no clinical significance between the different doses of dexamethasone and likelihood to reattend.

Conclusion This retrospective study emphasises that patients can safely be prescribed a lower dose of dexamethasone as a single dose for the management of croup and that local departments in the North-West should move to adopting 0.15 $\mathrm{mg} / \mathrm{kg}$ as their standard dose in their croup management guideline in line with current NICE guidelines.

\section{G251(P) WHICH CALCIUM IS BEST IN PAEDIATRIC RESICITATION? CALCIUM CHLORIDE VERSUS CALCIUM GLUCONATE FOR THE TREATMENT OF HYPOCALCEMIA IN ACUTE SETTING: AN EVIDENCE BASED SYSTEMATIC REVIEW}

${ }^{1,2} \mathrm{C}$ Kanaris, ${ }^{3} \mathrm{~A}$ Janjanam, ${ }^{3} \mathrm{~S}$ Palaniappan. ${ }^{1}$ Paediatric Intensive Care Unit, University Hospital North Midlands, Stoke-on-Trent, UK; ${ }^{2}$ North West and North Wales Transport Service, Manchester University FT, Warrington, UK; ${ }^{3}$ Paediatric Intensive Care Unit, Royal Manchester Children's Hospital, Manchester University FT, Manchester, UK

\subsection{6/archdischild-2020-rcpch.217}

Introduction/Objective A 2-year-old girl admitted with severe Group A streptococcal septic shock was resuscitated with blood products and polyinopressor support. She had persistent refractory hypocalcaemia despite multiple corrections with calcium gluconate which prompted us to do a systematic literature review for evidence regarding the usage of Calcium Gluconate and Calcium Chloride in the management of acute hypocalcemia, especially to ascertain if one confers a faster rise in iCa when used in an emergency. Secondary objectives we looked at included the pharmacokinetic basis, therapeutic efficacy, adverse event profile and physiological implications, again intending to clarify if there should be a clinical preference of one over the other in emergencies.

Methods Literature review using Cochrane library, Embase and Medline OVID interface. Papers published between January 1980 and October 2019

Results It is purported that Calcium Gluconate requires hepatic metabolism to release ionised Calcium, precluding its use in conditions of shock, cardiac arrest and hepatic impairment, presumably due to lower bioavailability and suboptimal biochemical effect. On the other hand, it is suggested that Calcium Chloride supposedly has rapid ionisation time, and could, in theory, provide ionised Calcium more quickly. This physiological reasoning is why historically, Calcium Chloride has been preferred in clinical situations where a rapid $\mathrm{iCa}$ is required like the scenarios mentioned above. However, the 